\title{
SCALAR O(N) MODEL AT FINITE TEMPERATURE - 2PI EFFECTIVE POTENTIAL IN DIFFERENT APPROXIMATIONS
}

\author{
JÜRGEN BAACKE and STEFAN MICHALSKI \\ Universität Dortmund \\ Institut für Physik, Otto-Hahn-Str. 4 \\ D-44221 Dortmund, Germany
}

\begin{abstract}
We calculate the two-particle irreducible (2PI) effective potential of the $O(N)$ linear sigma model in $1+1$ dimensions. The approximations we use are the nextto-leading order of a $1 / N$ expansion (for arbitrary $N$ ) and a kind of "resummed loop approximation" for $N=1$. We show that the effective potential of the $1 / N$ expansion is convex for $N=4$ and $N=10$ whereas it is not for the "loop" expansion and the case $N=1$ of the $1 / N$ expansion.
\end{abstract}

\section{Introduction}

Inspired by earlier analyses out of equilibrium and in thermal equilibrium ${ }^{1}$ we are interested in the phase structure of the $O(N)$ linear sigma model in different approximations. Since renormalization is quite a task in $3+1$ dimensions we first carry out this analysis in $1+1$ dimensions. For all details the reader is referred to a more comprehensive analysis published recently ${ }^{2}$.

\section{Basic Equations}

\subsection{Classical action}

We take the following classical action for the $O(N)$ linear sigma model with spontaneous symmetry breaking in $1+1$ dimensions

$\mathcal{S}[\Phi]=\int \mathrm{d}^{2} x \frac{1}{2}\left(\partial_{\mu} \vec{\Phi}\right)^{2}-\frac{\lambda}{4 N}\left(\vec{\Phi}^{2}-N v^{2}\right)^{2} \quad$ where $\quad \vec{\Phi}=\left(\Phi_{1}, \ldots, \Phi_{N}\right)$.

In order to consider finite temperature we use the Matsubara formalism where the Minkowskian integral over momentum space is transformed to a sum and an integral over spatial momenta. We will use the short-hand notation $T \sum_{\omega_{n}} \int \frac{\mathrm{dp}}{2 \pi} \equiv \oiint_{p}$. For the effective action we consider a homogeneous 
background condensate $\vec{\phi}=\langle\vec{\Phi}\rangle=(\phi, \phi, \ldots, \phi)$ which can be $O(N)$-rotated such that it points only in the 1 -direction $\vec{\phi}=(\sqrt{N} \phi, 0, \ldots, 0)$.

\subsection{PI effective action}

The two-particle irreducible (2PI) effective action ${ }^{4}$ reads

$$
\Gamma[\phi, G]=\mathcal{S}[\sqrt{N} \phi]+\frac{1}{2} \operatorname{Tr}\left(i \mathcal{D}^{-1} G-1\right)+\frac{i}{2} \ln \operatorname{det} G^{-1} D_{0}+\Gamma_{2}[G, \phi] .
$$

Here the two-point function $G$ is a $N \times N$ matrix which is $O(N)$ symmetric, $G=\operatorname{diag}\left[G_{\sigma}, G_{\pi}, \ldots, G_{\pi}\right]$, and $\mathcal{D}$ denotes the analogous matrix of classical Green functions with $i \mathcal{D}_{\sigma, \pi}^{-1}(k)=k^{2}-\lambda\left(f_{\sigma, \pi} \phi^{2}-v^{2}\right)$ and $f_{\sigma, \pi}=3,1$. The explicit form of all higher-order corrections denoted by $\Gamma_{2}[G, \phi]$ is related to the type of approximation used.

\subsubsection{Next-to-leading order of $1 / N$ expansion}

The $1 / N$ expansion is a systematic expansion of the effective action in powers of $1 / N$. The classical and one-loop part of the effective action is of leading order $\mathcal{O}(N)$ whereas all further contributions (except for the doublebubble graph) are of higher order. Here we will only take into account the contributions of next-to-leading order (NLO) of the $2 \mathrm{PI}-1 / N$ expansion $^{\mathrm{a}}$ Therefore, higher loop contributions are separated into three parts

$$
\Gamma_{2}[\phi, G]=\Gamma_{2}^{\mathrm{db}}[G]+\Gamma_{2}^{\text {pearls }}[G]+\Gamma_{2}^{\text {sunset }}[\phi, G] .
$$

The first term (so called double-bubble) contributes both at leading and next-to-leading order of $1 / N$ (cf. larger articles for details ${ }^{2,3}$ ) To sum up all contributions of next-to-leading order (NLO) we use the functional ${ }^{3}$

$$
\Gamma_{2}^{\text {pearls }}[G]=\frac{i}{2} \oiint_{p}\left\{\ln \left[1+i \frac{\lambda}{N} \operatorname{Tr} \mathcal{F}(p)\right]-i \frac{\lambda}{N} \operatorname{Tr} \mathcal{F}(p)\right\},
$$

where $\operatorname{Tr} \mathcal{F}$ is the trace of all fish graphs

$$
\operatorname{Tr} \mathcal{F}(p)=\mathcal{F}_{\sigma}(p)+(N-1) \mathcal{F}_{\pi}(p) \quad \text { with } \quad \mathcal{F}_{*}(p)=\oint_{p} G_{*}(k) G_{*}(k+p) .
$$

The subtracted graph in Eq. (3) is the NLO part of the double-bubble which is dealt with separately ${ }^{2}$.

${ }^{a}$ Due to resummation contributions of all powers of $1 / N$ contribute as well. Though the order is determined from the $2 \mathrm{PI}$ graphs of the action. 
The generalization of sunset diagrams in the effective action is achieved by cutting a sigma line of $\Gamma_{2}^{\text {pearls }}$ and pinning the two open legs to the background by multiplying by a factor of $N \phi^{2}$

$$
\Gamma_{2}^{\text {sunset }}[\phi, G]=i \lambda \phi^{2} \oiint_{p} G_{\sigma}(p) \frac{i \frac{\lambda}{N} \operatorname{Tr} \mathcal{F}(p)}{1+i \frac{\lambda}{N} \operatorname{Tr} \mathcal{F}(p)} .
$$

Expanding this in powers of $\mathcal{F}$ one finds graphs of the generalized sunset kind (see again our larger article ${ }^{2}$ for figures and more details).

\subsection{2. "Loop" expansion for $N=1$}

Since for $N=1$ the $1 / N$ expansion is somewhat meaningless, one could have the idea to improve a loop expansion by summing up all pearls and generalized sunset graphs as in Eqs. (3) and (5). We will call this "loop expansion" although this is not literally correct. We take into account the same graphs (topologically) as in the $1 / N$ expansion but with a combinatorical factor that disregards their order of $1 / N$. The respective expression for the resummation of pearls is (note the additional combinatorical factor)

$$
\Gamma_{2}^{\text {pearls }}[G]=\frac{i}{2} \oint_{p}\left\{\ln [1+3 i \lambda \mathcal{F}(p)]-i 3 \lambda \mathcal{F}(p)+3 \lambda^{2}[\mathcal{F}(p)]^{2}\right\} .
$$

The subtracted graphs in Eq. (6) have to be dealt with separately due to different combinatorical factors. The last term in Eq. (6) is the basketball graph which has a different topology than the graphs with more than two vertices. The sum of generalized sunset graphs is obtained in an analogous way to the $1 / N$ expression (5). It reads

$$
\Gamma_{2}^{\text {sunset }}[\phi, G]=i \lambda \phi^{2} \oint_{p} G_{\sigma}(p) \frac{3 i \lambda \mathcal{F}(p)}{1+3 i \lambda \mathcal{F}(p)}+6 \lambda^{2} \mathcal{F}(p) G(p) .
$$

\subsection{Dyson-Schwinger Equation}

In order to calculate the 1PI effective potential from the 2PI effective action we have to solve the Dyson-Schwinger equation $\delta \Gamma / \delta G=0$ for the 2-point function. Using the convention $i G_{*}^{-1}(p)=i \mathcal{D}_{*}^{-1}-\Sigma_{*}(p)$, where $*=\sigma$, $\pi$, we can express the Dyson-Schwinger equation in terms of the self-energy $\Sigma(p)$

$$
\begin{aligned}
& \Sigma_{\sigma}(p)=3 \frac{\lambda}{N} \mathcal{B}_{\sigma}+(N-1) \frac{\lambda}{N} \mathcal{B}_{\pi}-2 \frac{\delta}{\delta G_{\sigma}(p)}\left(\Gamma_{2}^{\text {pearls }}+\Gamma_{2}^{\text {sunset }}\right) \\
& \Sigma_{\pi}(p)=\frac{\lambda}{N} \mathcal{B}_{\sigma}+(N+1) \frac{\lambda}{N} \mathcal{B}_{\pi}-2 \frac{\delta}{\delta G_{\pi}(p)}\left(\Gamma_{2}^{\text {pearls }}+\Gamma_{2}^{\text {sunset }}\right) .
\end{aligned}
$$


The 1PI effective action is obtained by substituting a solution $G(\phi)$ of Eqs. (8) into $\Gamma[\phi, G]$. We will plot the 1PI effective potential that differs from that only by a total factor of volume times temperature.

\section{Numerical Results}

For a given temperature $T$ and different values of $\phi$ we numerically solve the Dyson-Schwinger equation (8) by iteration.

\subsection{Next-to-leading order of $2 P I 1 / N$ expansion}

We take here a value of $\lambda=0.5$ for the coupling constant and show results for $N=1, N=4$ and $N=10$. Figure 1 indicates that there is only one
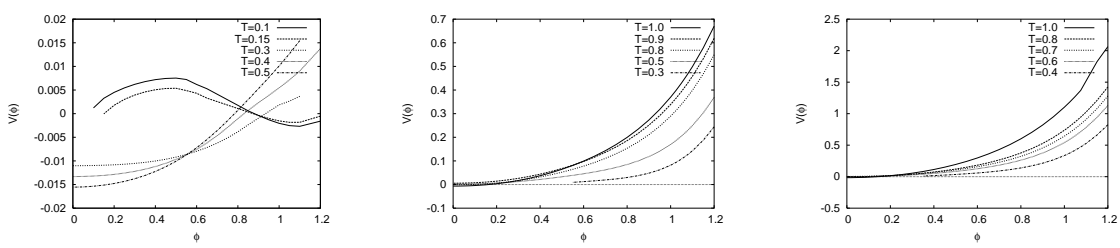

Figure 1. Shape of the effective potential in the $2 \mathrm{PI} 1 / N$ approximation at NLO. From left to right: $N=1$ and $\lambda=0.1$ (not normalized), $N=4$ and $\lambda=0.5, N=10$ and $\lambda=0.5$.

vacuum at $\phi=0$ for the considered temperatures and the cases $N=4$ and $N=10$. The potential for $N=1$ shows signs of a false vacuum which is actually not expected in a $1 / N$ expansion. Though for $N=1$ this expansions is obviously not meaningful. For the sake of direct comparison we mention - without showing a plot - that for $N=1$ and $\lambda=0.5$ the $1 / N$ potential is convex at temperatures $T=0.7$ and $T=0.8$, e.g., whereas it is not when using the "loop expansion" (see below and Fig. 2).

\section{2. "Loop expansion" for $N=1$}

We display the effective potential in the resummed "loop expansion" (cf. Eqs. (6) and (7)) in Fig. 2 at different temperatures and for three values of the coupling constant. The potential exhibits a typical structure with a false vacuum - a clear sign of a first-order phase transition. For higher temperatures, e.g. $T=1.2$ and $\lambda=1$ or $T=0.8$ and $\lambda=0.5$, the false vacuum has disappeared but a "relic" consisting of two inflection points remains. 

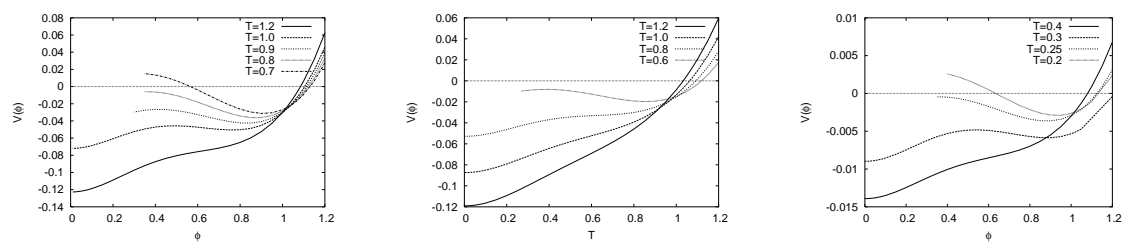

Figure 2. Effective potential of the "resummed loop expansion" at different temperatures for $\lambda=1, \lambda=0.5$ and $\lambda=0.1$.

\section{Conclusion and Outlook}

We have solved the Dyson-Schwinger equation to compute the effective potential of the $O(N)$ linear sigma model in $1+1$ dimensions both in a "resummed loop" expansion for $N=1$ and at NLO of a $1 / N$ expansion for arbitrary $N$. For $N=4$ and $N=10$ the effective potential is convex for all parameters we chose as expected from very old arguments ${ }^{5}$.

For $N=1$ we find (indications of) false vacua in both approximations. The $1 / N$ expansion seems to be meaningless for $N=1$ concerning the shape of the effective potential. For the "resummed loop expansion" one has to admit that this approximation only serves as an example of a non-systematic expansion and therefore the effective potential has a nonphysical shape. As stated above, further results can be found in a more comprehensive publication ${ }^{2}$.

\section{Acknowledgments}

S.M. thanks all the organizers of SEWM 2004 for a wonderful meeting in Helsinki. S.M. was supported by Deutsche Forschungsgemeinschaft as a member of Graduiertenkolleg 841.

\section{Bibliography}

1. J. BaAcke and S. Michalski, Phys. Rev. D 67, 085006 (2003) [arXiv:hep-ph/0210060]; Phys. Rev. D 65, 065019 (2002) [arXiv:hep-ph/0109137]; J. BAACKe and A. Heinen, Phys. Rev. D 68, 127702 (2003) [arXiv:hep-ph/0305220]. Phys. Rev. D 67, 105020 (2003) [arXiv:hep-ph/0212312].

2. J. BAACKE and S. MiChALSKI, arXiv:hep-ph/0407152 (to be published in PRD).

3. G. Aarts, D. Ahrensmeier, R. Baier, J. Berges and J. Serreau, Phys. Rev. D 66, 045008 (2002) [arXiv:hep-ph/0201308]; J. Berges, Nucl. Phys. A 699, 847 (2002) [arXiv:hep$\mathrm{ph} / 0105311]$.

4. see e.g. J. M. Cornwall, R. Jackiw and E. Tomboulis, Phys. Rev. D 10, 2428 (1974).

5. S. R. Coleman, "There Are No Goldstone Bosons In Two-Dimensions", Commun. Math. Phys. 31, 259 (1973). 